\title{
Management of Kawasaki disease in the British Isles
}

\author{
R Dhillon, L Newton, P T Rudd, S M Hall
}

\begin{abstract}
Kawasaki disease in the British Isles was surveyed by an active reporting scheme, based on all cases reported to the British Paediatric Surveillance Unit that were diagnosed between 1 January and 31 December 1990 . The study was prompted by the need to investigate the high case fatality rate of Kawasaki disease of $2 \%$ observed in 1988.
\end{abstract}

One hundred and sixty three patients were identified of whom six $(3 \cdot 7 \%)$ died. Forty five children $(28 \%)$ suffered cardiac complications of which $39(24 \%)$ were coronary artery abnormalities; five children were diagnosed at postmortem examination, and coronary artery abnormalities were detected by echocardiography in 34. One hundred and forty nine children (93\%) had echocardiography. High thrombocytosis, leucocytosis, duration of fever, and younger age were associated with the presence of coronary artery abnormalities. Erythrocyte sedimentation rate, sex, and the number of diagnostic criteria were not. One hundred and thirty three children $(87 \%)$ received aspirin. Ninety three children $(61 \%)$ received intravenous gammaglobulin (IVGG). Children were more likely to receive IVGG if they had thrombocytosis or typical Kawasaki disease. The incidence of coronary artery abnormalities was found to be similar in those treated with IVGG $(29 \%)$ and those untreated $(20 \%)$, including those treated within 10 days of onset. This may have reflected selection of the more serious cases to receive IVGG or that Kawasaki disease in the British Isles is a different illness to that experienced elsewhere. It may be, however, that IVGG is less effective in the treatment of British patients with Kawasaki disease than has been the experience in the United States and Japan. These observations emphasise the need for a therapeutic trial of treatment modalities for Kawasaki disease in the UK and the Republic of Ireland.

(Arch Dis Child 1993; 69: 631-638)

Bath Unit for Researc into Paediatrics, Royal United Hospital, Bath R Dhillon

P T Rudd

Public Health

Laboratory Service, Communicable Disease Surveillance Centre, 61 Colindale Avenue, London Avenue, Lon

NW9 5EO

S M Hall

Correspondence to: Dr Hall.

Accepted 1 September 1993

Kawasaki disease, an acute febrile illness of unknown aetiology mainly occurring in childhood, was first described in Japan in $1967 .{ }^{1}$ It has been recognised in the British Isles since the early 1970s. Although apparently a self limiting illness in most patients, Kawasaki disease is complicated by the development of coronary artery abnormalities in a proportion of cases. These may persist and cause ischaemic heart disease. They may also cause death, either in the acute phase of the illness, or months or even years later. Intravenous gammaglobulin (IVGG) has been shown to reduce the risk of coronary artery abnormalities if administered within 10 days of onset of Kawasaki disease. ${ }^{2}$

Epidemiological surveillance of Kawasaki disease in the British Isles, undertaken jointly by the British Paediatric Association and the Public Health Laboratory Service Communicable Disease Surveillance Centre, began in January $1983 .{ }^{3}$ A case fatality rate of $2 \%$ among reported cases in 1988 compared with $0 \cdot 1 \%$ in Japan in $1986^{4}$ prompted enhancement of surveillance over a 12 month period in order to collect detailed information on investigation and treatment of cases reported. The British case fatality rate was comparable with that seen in Japan before the introduction of treatment with IVGG, suggesting that some patients were not receiving IVGG - either at all, or within 10 days of onset; or that IVGG was less efficacious in patients with Kawasaki disease in the British Isles.

\section{Patients and methods}

Cases were recruited whose onset of Kawasaki disease was between 1 January and 31 December 1990. Ascertainment was via the British Paediatric Surveillance Unit (BPSU) 'active' reporting scheme. ${ }^{5}$ All consultant paediatricians throughout the UK and Ireland received a report card each month on which they were asked to indicate whether or not they had cared for a patient conforming to the standard criteria for Kawasaki disease (table 1) during the preceding calendar month. 'Typical' Kawasaki disease requires at least five of the six features to be present. However, cases were also accepted if: (a) there was echocardiographic or postmortem evidence of coronary artery abnormalities irrespective of the number of clinical criteria or (b) there were at least three criteria and the clinician was convinced of the

Table 1 Diagnostic criteria for Kawasaki disease: distribution in 163 patients reported in 1990

\begin{tabular}{lll}
\hline & & $\begin{array}{l}\text { No (\%) } \\
\text { of cases }\end{array}$ \\
\hline 1 & $\begin{array}{l}\text { Fever for more than five days } \\
2\end{array}$ & $146(90)^{\star}$ \\
3 & $\begin{array}{l}\text { Eolymorphema, indurative oedema, or } \\
\text { desquamation of hands and feet }\end{array}$ & $156(96)$ \\
4 & $143(88)$ \\
5 & Oral mucosal changes & $140(86)$ \\
6 & Bilateral conjunctival injection & $136(83)$ \\
\end{tabular}

^In an additional 13 cases duration of fever was unspecified $(n=4)$ or $<5$ days $(n=9)$. 
Table 2 Extract from consensus statement on the management of uncomplicated Kawasaki disease made by North American participants of the Third International Kawasaki Disease Symposium ${ }^{6}$

(1) Detection of cardiac involvement

Echocardiographic examination:

(A) as soon as diagnosis is suspected

(B) repeated at least at: 14-21 days, 60 days, and 12 months after onset

(2) Acute stage treatmen

Administer within 10 days of onset:

Aspirin $80-100 \mathrm{mg} / \mathrm{kg} /$ day in four divided doses until about the 14th illness day

IVGG $400 \mathrm{mg} / \mathrm{kg}$ once daily for four consecutive days

(3) Convalescent stage

(After 14th day of illness in afebrile patient)

Aspirin 3-5 mg/kg/day in a single dose to be discontinued 6-8 weeks after onset if no coronary abnormalities are detected on echocardiography

diagnosis and could find no more reasonable explanation other than Kawasaki disease for the clinical and laboratory findings.

Paediatricians who reported cases were invited to complete a proforma requesting details of clinical features, laboratory results, cardiac investigations (details of echocardiographic examination as well as electrocardiography (ECG) findings were specifically sought), and treatment. Information on outcome at the time of questionnaire completion was also requested. Analyses were undertaken such that management could be compared with that outlined in a consensus statement made in 1989 by North American attendees of the Third International Kawasaki Disease Symposium $^{6}$ (table 2 ).

\section{STATISTICAL METHODS}

The Mann-Whitney U test was used for comparisons of age distributions between patients with and without coronary artery abnormalities, and between those treated and untreated with IVGG. Haematological variables were compared between these groups using the unpaired $t$ test. Yates's corrected $\chi^{2}$ test was used in the analysis of the incidence of coronary artery abnormalities within different treatment groups and other categorical data.

\section{Results}

The average monthly BPSU report card return rate in 1990 was $90 \%$. A total of 182 reports of Kawasaki disease were received and a completed proforma was provided for $166(91 \%)$ of these. Three patients were excluded because they had fewer than three of the diagnostic criteria and no coronary artery involvement, leaving 163 for analysis (table 1). The reported incidence per $10^{5}$ children under 5 years old in the British Isles was $3 \cdot 4$. The interval between date of onset of first symptom and questionnaire completion ranged between 13 days and 18 months (median 10 weeks).

One hundred and thirty one children ( $80 \%)$ had typical Kawasaki disease with five or six criteria. Of the remaining 32, 22 had four criteria and 10 had three. Ninety six were boys and 67 were girls (ratio 1·4:1). Ages ranged from 5 weeks to 15 years with a median of 24.5 months. Eighty three per cent were less than 5 years and $29 \%$ were less than 12 months old. One hundred and thirty seven $(86 \%)$ were white - a relative under-representation compared with the British population under 15 $(90 \%)$ as a whole; seven $(4 \%)$ were black and five $(3 \%)$ were oriental (these ethnic groups together account for about $2 \%$ of the base population).

The interval between onset of the first symptom and diagnosis of Kawasaki disease ranged between one and 304 days (median nine). One child had had a febrile illness at the age of 2 months and the retrospective diagnosis of Kawasaki disease was made 304 days after probable onset. Extensive coronary artery aneurysms were found at necropsy after the child had died suddenly at age 12 months. There were 25 patients diagnosed with Kawasaki disease four or fewer days after onset of first symptom, even though five days' fever is one of the diagnostic criteria. All except four of these had five or six of the classic criteria and all except three (one of whom had coronary artery abnormalities) went on to have a fever lasting at least five days. Three of the total five oriental children in the series were in this 'early diagnosis group'; one of them, a Chinese infant, was diagnosed on the second day after onset, when five criteria were present.

\section{CARDIAC AND OTHER SEQUELAE}

In this study population of 163 patients, 45 children (28\%) had evidence of cardiac complications: 39 (24\%) had coronary artery abnormalities; one child each had congestive cardiac failure and mitral regurgitation; four had a pericardial effusion. Of the 39 children with coronary artery abnormalities, 22 had coronary artery aneurysms and 17 had coronary artery dilatation; 34 were diagnosed by echocardiography and five at postmortem examination. Six of these 39 patients died - all were white boys with ages at onset ranging from 2 to 24 months. In only one was the diagnosis made in life and this was the only child of these six to receive specific treatment for Kawasaki disease. Three of them had 'atypical' Kawasaki disease with four or fewer diagnostic criteria.

Children with coronary artery abnormalities were significantly younger and had a longer fever duration than those without, but sex and the number of diagnostic criteria were not significantly associated with coronary artery abnormalities (table 3 ).

Sixty nine children (42\%) had a total 71 non-cardiac complications, of which the most common were abdominal or urogenital complaints (pain, diarrhoea and vomiting, gallbladder hydrops, sterile pyuria) and arthralgia/ arthritis. There were no deaths other than those associated with coronary artery abnormalities, thus the overall fatality rate was $6 / 163(3 \cdot 7 \%)$. Outcome of the survivors one year after onset is the subject of a separate study.

LABORATORY INVESTIGATIONS

Microbiological findings consistent with current and recent infection were reported in 33 
$(21 \%)$ of 155 patients. There was no consistency except for group A streptococci which were cultured in eight cases, among whom seven had five or six criteria for Kawasaki disease and two had coronary artery abnormalities. A further five patients had an increased antistreptolysin 0 titre including four exhibiting at least five criteria for Kawasaki disease and one who had coronary artery abnormalities.

Haematological findings are shown in table 3. There was a significant association between the presence of coronary artery abnormalities and the degree both of peak thrombocytosis and peak leucocytosis, but not erythrocyte sedimentation rate.

\section{CARDIAC INVESTIGATIONS}

One hundred and twenty nine (84\%) of 153 patients with this information had ECG on at least one occasion. Eighteen of them (14\%) had at least one abnormality, including: $T$ wave flattening or inversion (five); sinus tachycardia (three); ST segment depression or elevation (four); abnormal $\mathrm{Q}$ waves (three) and miscellaneous (four): first degree A-V block; right axis deviation; bundle branch block (laterality not specified); left ventricular 'strain'.

One hundred and forty nine (93\%) of the 160 children with information had at least one echocardiogram, of whom 59 (37\% of the total) had at least two within three weeks of diagnosis. Of 117 patients with information, the interval from diagnosis of Kawasaki disease to first echo ranged from 0-56 days with a median of three days. Among the 11 cases in whom echocardiography was not undertaken, there were five for whom it was felt to be 'not clinically indicated'; in one a lack of local facilities was given as the reason, and five patients died before echocardiography could be undertaken.

Of the 33 children who had both echo evidence of coronary artery abnormalities and ECG performed, the ECG was abnormal in only $10(30 \%)$. Among the remaining 23 there were 10 patients for whom there were sufficient data to show that the normal ECG had been performed contemporaneously with the abnormal echocardiogram. There was insufficient information to determine this in the remainder. The other eight children with abnormalities on ECG all had normal echocardiograms reported.

\section{TREATMENT}

Details of treatment were provided for 153 patients. One hundred and thirty three $(87 \%)$ received aspirin, of whom 71 (46\% of the whole group) received it in a biphasic dosage regimen (high anti-inflammatory dose followed by low antiplatelet dose; table 2). The dose in the first or only phase ranged from $2-183 \mathrm{mg} / \mathrm{kg}$ (mean (SD) $56.6(38.2)$ ) while that for the second phase ranged from $2-75$ $\mathrm{mg} / \mathrm{kg}$ (mean (SD) $13.2(15 \cdot 1)$ ). Duration of treatment for the first dose ranged from two to 135 days (median 22). The duration of the second dosage could not be assessed because of the relatively short follow up interval in this study. The interval between onset of first symptom and start of aspirin treatment ranged from two to 41 days (median nine).

Ninety three $(61 \%)$ of 153 children with treatment information received IVGG. The daily dose ranged from 33-1000 $\mathrm{mg} / \mathrm{kg}$ (median $400 \mathrm{mg} / \mathrm{kg}$ ) administered over periods ranging from one to six days (median four). The interval from first symptom to treatment ranged from one to 27 days (median nine days). The one day interval was in the Chinese patient described earlier who was diagnosed very early. It was, however, noteworthy that four (all typical) of the 25 patients diagnosed before five days of illness were not given IVGG.

Ninety two of 153 children $(60 \%)$ received both aspirin and IVGG (figure). Among these there were 11 ( $7 \%$ of the total 153 with information) who had received the consensus group recommended treatment of high dose aspirin $(80-100 \mathrm{mg} / \mathrm{kg})$, plus high dose IVGG for four days within 10 days of onset (table 2).

Fifteen children received dipyridamole, all of them in addition to aspirin, and 13 had also received IVGG; six of these 15 had coronary artery abnormalities. One other child was given a short course of prednisolone; about half of the patients received a variety of other medications, mainly antibiotics.

CORONARY ARTERY ABNORMALITIES AND IVGG IVGG is believed to have a preventive effect on the development of coronary artery abnormalities so this was examined in more detail. Overall, the incidence of coronary artery abnormalities in children treated with IVGG, $27 / 93(29 \%)$, was similar to that, $12 / 60(20 \%)$ in those untreated $(p=0 \cdot 29)$. The incidence $(15 / 55,27 \%)$ of coronary artery abnormalities in children who received IVGG within 10 days of onset (includes $12(22 \%)$ who received 400 $\mathrm{mg} / \mathrm{kg}$ for four days) was also not significantly different from those untreated. However, the incidence of coronary artery abnormalities, $12 / 33(36 \%)$, among those who received it later, was significantly higher than the untreated group (table 4).

As this survey was a description of actual practice rather than a therapeutic trial, the two groups of patients, those treated and those

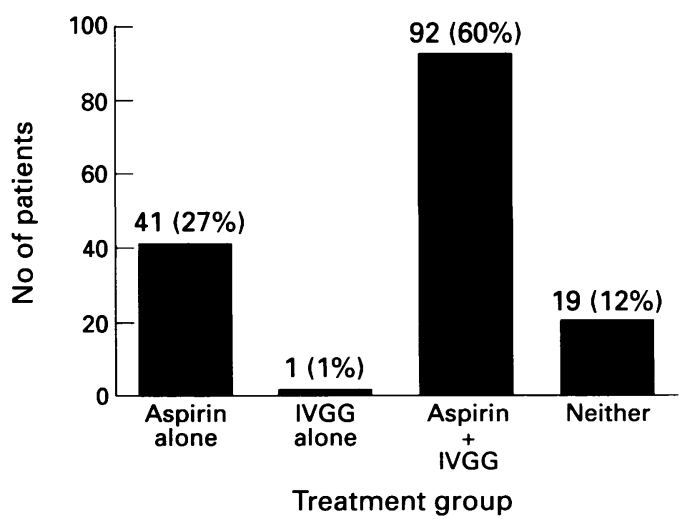

Kawasaki disease 1990: treatment in 153 patients (treatment was unknown in 10 patients). 
Table 3 Clinical and epidemiological characteristics

\begin{tabular}{|c|c|c|c|c|c|}
\hline Variable & $\begin{array}{l}\text { Whole } \\
\text { group } \\
(n=163)\end{array}$ & $\begin{array}{l}\text { Cononary artery } \\
\text { abnormalities present } \\
(n=39)\end{array}$ & $\begin{array}{l}\text { No coronary artery } \\
\text { abnormalities } \\
(n=124)\end{array}$ & $p$ Value $^{\star}$ & $\begin{array}{l}\text { Confidence } \\
\text { interval } \\
(95 \%) \dagger\end{array}$ \\
\hline $\begin{array}{l}\text { Median age (months) } \\
\text { Sex ratio (\% male) } \\
\text { Mean (SD) peak platelets }\left(\times 10^{9} /\right) \\
\text { Mean (SD) peak white cell count }\end{array}$ & $\begin{array}{l}24 \cdot 5 \\
58 \\
796(249)\end{array}$ & $\begin{array}{l}11 \\
56 \\
905(184)\end{array}$ & $\begin{array}{l}33 \\
59 \\
759(258)\end{array}$ & $\begin{array}{l}0.0001 \\
0.79 \\
0.002\end{array}$ & $\begin{array}{l}0.154 \text { to } 0.479 \\
-0.203 \text { to } 0.154 \\
53.8 \text { to } 238\end{array}$ \\
\hline $\begin{array}{l}\left(\times 10^{9} \Lambda\right) \\
\text { Mean }(S D) \text { peak erythrocyte }\end{array}$ & $22(8)$ & $25 \cdot 2(9)$ & $21 \cdot 5(7)$ & 0.02 & 0.61 to 6.78 \\
\hline $\begin{array}{l}\text { sedimentation rate }(\mathrm{mm} / \mathrm{h}) \\
\text { Mean (SD) fever duration (days) } \\
\text { No (\%) criteria }\end{array}$ & $\begin{array}{l}81(33) \\
12 \cdot 8(9)\end{array}$ & $\begin{array}{l}84 \cdot 8(29) \\
16 \cdot 9(12)\end{array}$ & $\begin{array}{l}80 \cdot 3(30) \\
11 \cdot 5(7)\end{array}$ & $\begin{array}{l}0.56 \\
0.0006\end{array}$ & $\begin{array}{r}-10 \cdot 8 \text { to } 19 \cdot 8 \\
2 \cdot 27 \text { to } 8 \cdot 53\end{array}$ \\
\hline$\leqslant 4$ & $\begin{array}{r}131(80) \\
32\end{array}$ & $\begin{array}{c}32(82) \\
7\end{array}$ & $\begin{array}{l}99(80) \\
25\end{array}$ & 0.66 & -0.11 to 0.17 \\
\hline
\end{tabular}

*Coronary artery abnormalities present $v$ absent.

†Confidence interval of the differences.

untreated with IVGG, were compared for number of diagnostic criteria and for those features shown in table 3 which might predispose them both to being at risk of coronary artery abnormalities and to being more likely to be given treatment (table 4). Children who received IVGG early, had higher peak platelet counts than those untreated and were more likely to have typical Kawasaki disease. Similar differences were observed between the late IVGG and the untreated groups and these patients were also significantly younger.

\section{Discussion}

The reported incidence of Kawasaki disease in the British Isles (3.4 per 100000 children under 5 years in 1990) was much lower than that in Japan (172.2 per 100000 children under 5 years in $1986^{4}$ ). The incidence was, however, slightly higher than has been recorded in the USA as derived from a passive surveillance scheme. ${ }^{7}$ The age, sex, and ethnic distribution were similar to those described in US series with a higher preponderance of oriental and black children. ${ }^{8}$ The $3 \cdot 7 \%$ case fatality rate observed in the Kawasaki disease patients in 1990 was high compared with that in Japan $\left(0 \cdot 1 \%\right.$ in $\left.1986^{4}\right)$. All of those children who died had coronary artery abnormalities and the incidence of coronary artery abnormalities overall was $24 \%$, which is comparable with that seen in untreated Japanese and American children with Kawasaki disease. ${ }^{2} 910$

Coronary artery abnormalities were more likely in young children in this study and in those with marked thrombocytosis and leucocytosis, as has been shown by other workers. ${ }^{11} 12$ Neither a raised erythrocyte sedimentation rate nor sex were associated with the presence of coronary artery abnormalities and this was also the finding in another British series, ${ }^{12}$ but contrasts with the observations of workers in Japan ${ }^{13}$ and the USA. ${ }^{11}$ Duration of fever was also related to the development of coronary artery abnormalities in the 1990 British patients. This has been documented as a predictor in some studies ${ }^{11} 1314$ but not others. ${ }^{15}$

The 1990 patients also demonstrated the poor sensitivity of the standard case definition of Kawasaki disease. This is useful for epidemiological surveillance and to facilitate comparisons with other series, but clinicians should be aware that it underascertains the now well described atypical Kawasaki disease in which patients exhibit fewer than five criteria, but nevertheless develop coronary artery abnormalities. ${ }^{6}$ Such cases accounted for $18 \%$ of the 39 patients with coronary artery abnormalities in 1990. Acceptance of cases of 'Kawasaki disease' not conforming to the standard definition, but considered by the clinician to be the most likely diagnosis, probably diluted this series with non-Kawasaki disease patients; the high coronary artery abnormalities rate was, therefore, all the more striking.

A consensus group of North American participants of the Third International Kawasaki Disease Symposium has recommended that all children with suspected Kawasaki disease should have serial echocardiography including two examinations in the first three weeks after onset (table 2) ${ }^{6}$ This would optimise recognition of coronary artery abnormalities and guide both acute and long term management (for example, the duration of usage of antiplatelet agents); moreover, it would provide prognostic information for the

Table 4 Coronary artery abnormalities and the use of IVGG

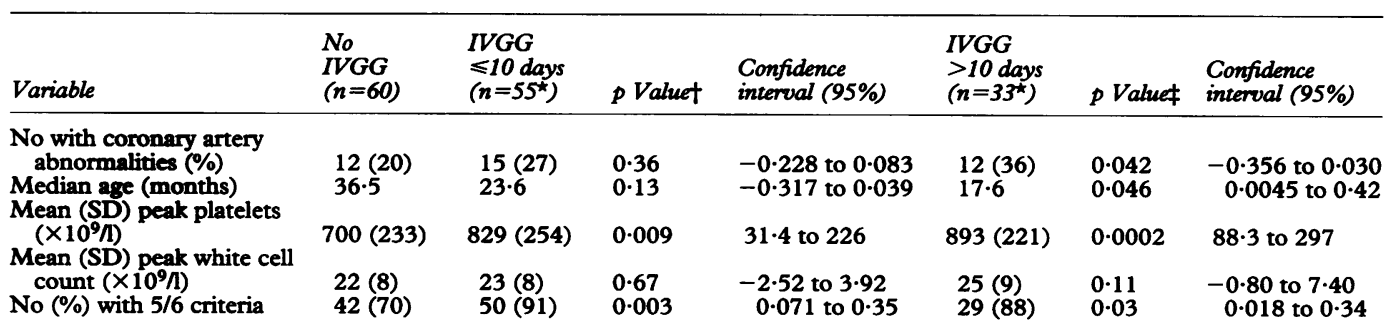

*In five patients intervals from onset of symptoms to treatment with IVGG was unknown.

+ No IVGG $v$ IVGG $\leqslant 10$ days.

$\ddagger$ No IVGG $v$ IVGG $>10$ days. 
parents. There were $11(7 \%)$ children among the 1990 British cases in whom no echocardiography was performed - a lack of local facilities was given as the reason in only one case; furthermore, among those who did receive echocardiography, less than a half had two examinations in the three weeks after onset.

The relatively small proportion, $14 \%$, of patients in whom abnormal ECG was reported was surprising, because other series describe proportions as high as $80 \% .{ }^{15}$ This may reflect the unselected nature of the 1990 study population that included patients at both ends of the range of severity and probably some who did not have Kawasaki disease. While some workers have found good correlation between ECG and echo changes, ${ }^{16}$ others suggest that ECG is a poor predictor of coronary artery abnormalities because of lack of specificity. ${ }^{15}$ We drew the same conclusion, but for reasons of sensitivity: it was reported as normal in at least 10 patients with contemporaneous evidence of coronary artery abnormalities on an echocardiogram. So while examination by ECG has a role in the evaluation of the pancarditis of Kawasaki disease, it should always be as an adjunct to echocardiography.

It is noteworthy that, despite the high coronary artery abnormalities rate, over a half of the children among the 1990 British Isles cases had received both aspirin and IVGG. Several controlled studies have shown a beneficial effect of IVGG given within 10 days of onset in the prevention of coronary artery abnormalities. $^{29} 10$ In 1986 Newburger et al demonstrated prospectively a coronary artery abnormalities incidence of $8 \%$ in the IVGG (400 $\mathrm{mg} / \mathrm{kg}$ daily for four days) group against $23 \%$ in the control group at two weeks after onset. The respective incidence of coronary artery abnormalities at seven weeks was $4 \%$ against $18 \% .^{2}$ More recently, the same workers have shown that a single large dose of $2 \mathrm{~g} / \mathrm{kg}$ administered within 10 days of onset and infused over 10 hours is even more effective (4.6\% coronary artery abnormalities at two weeks) than four infusions $(9 \cdot 1 \%) .{ }^{17}$ The efficacy of IVGG administered after 10 days is unknown.

In our study, $29 \%$ of all children receiving IVGG had coronary artery abnormalities, compared with $20 \%$ left untreated. Of particular interest, the proportion of children with coronary artery abnormalities was still $27 \%$ in those receiving IVGG within 10 days of onset of symptoms. Children receiving IVGG were more likely to be typical cases of Kawasaki disease and to have higher platelet counts than those in the untreated group. Those treated after 10 days were also significantly younger. It is, therefore, possible that the IVGG treated group was biased towards having more severe Kawasaki disease, because previous work has suggested that young age and thrombocytosis are adverse factors in the development of coronary artery abnormalities. ${ }^{11}$ Nevertheless, it is unlikely that these cases, especially the early group, were markedly more severe and typical than the patients recruited under stringent guidelines in the recent US IVGG trials $^{2} 17$ (which in any case did not relate treatment failure to specific antecedent risk factors). Furthermore, we collected information on peak platelet counts, not admission values: as thrombocytosis typically occurs in the second or third week of the illness it is unlikely that this biased the early treatment group.

Another possible cause of bias might have been preferential treatment of patients with established coronary artery abnormalities. Unfortunately, recording of date of the first abnormal echocardiogram was not often completed on the study proforma. This information was, however, discernible in 10 patients with coronary artery abnormalities; in eight of them the first abnormal echo occurred between 15 and 31 days after onset of first symptom; five of these eight had been given IVGG within the first 10 days after onset. The two other patients had echo changes reported at four days after onset and both of these were given IVGG at this stage. In general, however, studies of the natural history of Kawasaki disease suggest that early onset coronary artery abnormalities are unusual, with most becoming evident after 2-3 weeks. It seems unlikely (although we cannot rule it out) that the early treatment group would have been seriously biased by an excess of these unusual cases. It could, however, explain the poor outcome of the late treatment group.

Our retrospective analysis was, therefore, unable to demonstrate that IVGG protected British children from coronary artery abnormalities. A recently published analysis performed retrospectively on Japanese nationwide survey data collected over a six and a half year period gave similar results, with cardiac sequelae being more prevalent in those children who received IVGG. ${ }^{18}$

There are several possible reasons for this observation in the British Isles. First, there may have been the selection bias described above (this explanation was also suggested by the Japanese workers ${ }^{18}$ ). However, it is still difficult to explain the high overall incidence of coronary artery abnormalities, comparable with that seen in untreated children from Japan and the USA.

Second, Kawasaki disease in the British Isles may have a different aetiology from that experienced elsewhere. This comment has to be speculative, but it is made in the context of ignorance as to the cause of Kawasaki disease. ${ }^{6}$ In this study the patients included 13 children with evidence of streptococcal infection (three of whom had coronary artery abnormalities). If these 13 children are excluded from the analysis, 24 of $87(28 \%)$ IVGG treated children had coronary artery abnormalities compared with 12 of $54(22 \%)$ untreated children. The trend seen earlier with the full sample is unchanged.

Third, the precise mechanism of action of IVGG remains unclear, but there may be qualitative differences in IVGG according to the population from which the preparation is derived and the production process employed. ${ }^{19}$ The preparation most commonly used in Britain is harvested from unpaid 
donors from Switzerland, Germany, and the USA. A difference in IVGG preparation may therefore have contributed to the higher incidence of coronary artery abnormalities in the British IVGG treated group compared with the North American and Japanese patients.

Our survey data were derived from an enhanced population based surveillance scheme, not a therapeutic trial; echocardiographic findings, for example, were not subject to standardised interpretation. Nevertheless, they point to what may be a real difference from the North American and Japanese experience. It cannot be assumed that IVGG is universally an effective treatment - it may be beneficial in the management of some children with Kawasaki disease, but not in others. In spite of the overall finding with regard to coronary artery abnormalities many paediatricians in our survey commented on the rapid and dramatic symptomatic relief brought about by IVGG.

In the meantime, therefore, because there is convincing evidence both from Japan and the USA that treatment of Kawasaki disease patients in those countries in the first 10 days after onset with IVGG is effective 291017 we recommend its continued use in this country, but also that further research be undertaken formally to establish its efficacy in British patients with Kawasaki disease. This should also determine whether the consensus group guidelines on management of Kawasaki disease (table 2) are appropriate and practical in the UK and the Republic of Ireland (including an evaluation of the recommended echocardiography protocol, the efficacy of IVGG given after 10 days and that of different aspirin dosage regimens). Our survey suggested that only a proportion of patients were investigated (40\%) and treated (7\%) precisely as set out in these guidelines. Clearly these factors, especially use of an inappropriately low dosage of IVGG, might also explain the poor outcome. We did, however, experience difficulty in assessing dosage, both of IVGG and of aspirin, in a number of cases for whom total daily dose rather than $\mathrm{mg} / \mathrm{kg}$ was given.

The role of other therapeutic agents also requires assessment. Dipyridamole, for example, is usually recommended only for children with Kawasaki disease complicated by persistent aneurysms; nevertheless, 15 children were given this agent in 1990 and over half of them had no coronary artery abnormalities.

Reports of cases of cardiac disease in adults associated with Kawasaki disease in childhood are on the increase ${ }^{20}$ It is, therefore, important both that optimal management regimens are defined as soon as possible and that there is more widespread professional education in early recognition of this uncommon, but important childhood disorder that is readily confused with common exanthema such as measles and scarlet fever. Judging from our observation that half of the patients were not diagnosed with Kawasaki disease until 10 days or more after onset, there appears to be a need for improved diagnostic awareness both at primary and secondary care level.
In conclusion, there was a high rate of mortality and morbidity among reported cases of Kawasaki disease in the British Isles in 1990 when compared with patients treated with IVGG in Japan and the USA. Our survey suggests that there were at least three causes: some patients were untreated; some were treated but less than optimally when compared with published guidelines; finally, there was evidence that IVGG may be less effective for Kawasaki disease in British patients. These observations about potentially preventable childhood mortality and morbidity require more detailed exploration, especially as the preliminary findings of the Kawasaki disease surveillance scheme in the British Isles suggest that the proportion of cases complicated by coronary artery abnormalities showed little change between 1987 (29\%) and 1992 (20\%) (S Hall, unpublished data).

We wish to thank all paediatricians who reported cases and completed proformas. Without their help this study would not have been possible. We also thank Nazila Soltanpoor and Jane Bruce for statistical support. We acknowledge the generous support given to the BPSU by the Medical Research Fund of Child gicen Avon Rubber for their support for the Bath Unit for Research into Paediatrics.

1 Kawasaki T. Acute febrile mucocutaneous lymph node syndrome: clinical observations of 50 cases. Fapanese fournal of Allergy (Arerugi) 1967; 16: 178-222.

2 Newburger JW, Takahashi M, Burns JC, et al. The treatment of Kawasaki syndrome with intravenous gammaglobulin. N Engl f Med 1986; 315: 341-7.

3 Hall SM. Surveillance of Kawasaki disease in the British Isles. Arch Dis Child 1989; 64: 121-8.

4 Kawasaki T. Kawasaki disease. Asian Medical fournal 1989; 32: 497-506.

5 Hall SM, Glickman M. The British Paediatric Surveillance Unit. Arch Dis Child 1988; 63: 344

6 Shulman ST, Bass JL, Bierman F, et al. Management of Kawasaki syndrome: a consensus statement prepared by North American participants of the Third Intermational Kawasaki Disease Symposium, Tokyo, Japan, December 1988. Pediatr Infect Dis $\mathcal{f} 1989$; 8: 663-7.

7 Mason WH, Schneider T, Takahashi M. The epidemiology and etiology of Kawasaki disease. Cardiology in the Young 1991; 1: 196-205.

8 Newburger JW, Burns JC. Kawasaki syndrome. Cardiol Clin 1989; 7: 453-65.

9 Furusho K, Kamiya T, Nakano H, et al. High dose intravenous gammaglobulin for Kawasaki disease. Lancet 1984; ii: $1055-8$.

10 Nagashima $M$, Matsushima $M$, Matsuoka $H$, Ogawa $A$, Okumwa N. High dose gammaglobulin therapy for Kawasaki disease. $\mathcal{F}$ Pediatr 1987; 110: 710-2.

11 Ichida F, Fatica NS, Engle MA, et al. Coronary artery involvement in Kawasaki syndrome in Manhattan, New York: risk factors and role of aspirin. Pediatrics 1987: 80: 828-35.

12 Tizard EJ, Suzuki A, Levin M, Dillon MJ. Clinical aspects of 100 patients with Kawasaki disease. Arch Dis Child 1991; 66: 185-8.

13 Asai T. Evaluation method for the degree of seriousness in Kawasaki disease. Acta Paediatr $f_{p n}$ 1983; 25: 170-5.

14 Koren G, Rose V, Lavi S, et al. Probable efficacy of high dose salicylates in reducing coronary involvement in Kawasaki disease. $\mathcal{F A M A}$ 1985; 254: 767-9.

15 Hiew TW, Cheng HK. ECG abnormalities in Kawasaki disease and their value in predicting coronary artery aneurysms. Singapore Med f 1992; 33: 262-7.

16 Ichida F, Fatica NS, O'Loughlin JE, Snyder MS, Ehlers KH, Engle MA. Correlation of electrocardiographic and echocardiographic changes in Kawasaki syndrome. $\mathrm{Am}$ Heart $\mathcal{f} 1988$; 116: 812-9.

17 Newburger JW, Takahashi M, Beiser AS, et al. A single intravenous infusion of gamma globulin as compared with intravenous infusion of gamma globulin as compared with four infusions in the treatment of acute

18 Nakamura $Y$, Fujita $Y$, Nagai $M$, et al. Cardiac sequelae of Kawasaki disease in Japan: statistical analysis. Pediatrics 1991; 88: 1144-7

19 Greenbaum BH. Differences in immunoglobulin preparations for intravenous use: a comparison of six products. Am $\mathcal{F}$ Pediatr Hematol Oncol 1990; 12: 490-6.

20 Kato H, Inoue O, Kawasaki T, Fugiwara J, Watanabe T, Toshima $\mathbf{H}$. Adult coronary artery disease probably due to childhood Kawasaki disease. Lancet 1992; 340: 1127-9. 